\title{
Free word associations of children and adults'
}

JOAN WERTHEIM ${ }^{2}$ AND P. JAMES GEIWITZ 3

UNIVERSITY OF MICHIGAN

Free word associations of children and adults are compared to test the theory of three phases in intellectual development. Children(ages 3-4) gave significantly more "primitive" associations to 22 common nouns; adults (ages 19-24) evidenced a greater tendency to use abstract or "logical" relationships. Both groups gave an approximately equal number of the intermediate "physical" response. Implications for the general theory and the relationship to language acquisition are discussed.

Current theories of intellectual development, strongly influenced by Piaget, typically describe three levels of conceptual orientation (cf. Watson, 1959). The first level might be termed "primitive" since words are used in socially meaningless and/or uniquely personal ways. The older child functions at an intermediate or "physical" level; he understands and organizes words on the basis of physical or infralogical characteristics, e.g., their attributes and functions. Finally, the adult level is marked by "logical" or abstract relations with roots in the formal grammatical mules of our culture.

This study tests the general theory by analyzing the differences among these three levels in free word associations (FWA) of children and adults.

Method

Two groups of Ss provided the independent variable of age: (1) 12 children from predominantly middleclass nursery schools, 6 of age 3 and 6 of age 4 ; (2) 12 students at the University of Michigan, ranging in age from 19 to 24 . Both groups contained an equal number of males and females.

The stimulus words used to elicit FWA were 22 common nouns (Riegel, Riegel, Smith, \& Quarterman, 1964): table, man, house, butterfly, chair, river, window, carpet, girl, cabbage, stomach, bread, boy, cottage, head, child, city, doctor, bed, moon, cheese, and street. Responses were classified, independently by two coders, into 20 categories adapted from Riegel et al (1964) and from Woodworth (1938). The 20 differentiated relationships of FWA to noun-stimulus grouped themselves, both by content and by empirical trends, into the following more inclusive categories (with absorbed classifications in parentheses): ${ }^{4}$

I. Logical (Superordinate; subordinate; coordinate, similar, or contrast)

IIA. Physical-structure (Whole; part; location)

IIB. Physical-contingency (Activities by stimulus; activities done to or with stimulus; qualities)

IIC. Physical-contemporaneity (commonly related to stimulus in experience, but not I or IIA, B)
IIIA. Primitive-subjective (personal or proper names; evaluation; emotion)

IIIB. Primitive-unrelated ("triggered" responses; no common relation)

IIIC. Primitive-form-determined (clang associations; completions)

IIID. Primitive-no response ("I don't know"; no response; stimulus repeated)

Each $\mathrm{S}$ was tested individually. Four practice stimuli were administered first; after S's sample responses, E approved and, with child Ss only, noted other possible replies designed to vary in parts-of-speech. Then the 22 experimental words were randomly presented with a 20-sec. limit for responding. With the child Ss, the stimuli were phrased, "If I say (stimulus) to you, what would you say to me?"'

\section{Results}

A total of 528 responses were coded into the 20 separate categories with an intercoder percentgage agreement of $89 \%$. For the three major categories (I, II, III), percentage agreement reached 95\%. Disagreements were discussed until consensus was obtained.

Table 1 gives the results by age and sex for the groupings described above. Although some intriguing possibilities exist within subclass, the major trends are clearly in the major categories summarized in Table 2. The average child exhibits many more primitive responses while the average adult produces more of the logical type $\left(\mathrm{X}^{2}=12.39, \mathrm{p}<.01\right)$. Very little difference exists in the physical class.

Table 1. Frequency of FWA in Each Class

\begin{tabular}{lrrrrrrrrr} 
& 1 & IIA & IIB & IIC & IIA & IIB & IIC & IIID \\
\hline Age 3 & & & & & & & & \\
$\quad$ Male $(N=3)$ & 2 & 4 & 16 & 6 & 1 & 16 & 19 & 2 \\
$\quad$ Female $(N=3)$ & 6 & 7 & 5 & 9 & 1 & 31 & 3 & 4 \\
Age 4 & & & & & & & & \\
$\quad$ Male $(N=3)$ & 17 & 7 & 6 & 9 & 4 & 18 & 1 & 4 \\
$\quad$ Female $(N=3)$ & 6 & 8 & 5 & 12 & 4 & 26 & 2 & 3 \\
Adult & & & & & & & & \\
$\quad$ Male $(N=6)$ & 70 & 17 & 19 & 19 & 0 & 3 & 4 & 0 \\
$\quad$ Female $(N=6)$ & 55 & 21 & 24 & 19 & 3 & 1 & 9 & 0 \\
Totals & & & & & & & & \\
$\quad$ Children $(N=12)$ & 31 & 26 & 32 & 36 & 10 & 91 & 25 & 13 \\
$\quad$ Adults $(N=12)$ & 125 & 38 & 43 & 38 & 3 & 4 & 13 & 0 \\
\hline$\quad$
\end{tabular}

Table 2. Average Frequencies for Major Categories

\begin{tabular}{lccc} 
& Logical (I) & Physical (II) & Primitive (III) \\
\hline Child & 2.58 & 7.83 & 11.58 \\
Adult & 10.42 & 9.92 & 1.67 \\
\hline
\end{tabular}




\section{Discussion}

The results generally support the commonly presented theory of the development in concept orientation. The very young child-in this case, a mere year or two beyond the first word-tends to exhibit primitive relationships between stimuli and FWA. His associations show more personal and more sound-determined orientations, both of which are relatively less sociocentric than other types. The high frequency of unrelated responses is similarly indicative of the lack of formal organization in the child's orientation. Children, in addition, are more likely to find themselves unable to produce any association to a word.

Adults, on the other hand, give considerably more responses that are "logical"; i.e., involving abstract relationships prescribed by formal language rules. Such a finding suggests that the development of concept orientation closely parallels the course of language acquisition, an assumption underlying the current trend in purely linguistic coding schemes (Brown \& Berko, 1960; Ervin, 1961; Entwisle, Forsyth, \& Muuss, 1964).

The fact that children, if their response was not primitive, usually gave physical associates is also of interest. We had conceived the physical class as one primarily developed in the course of everyday observa- tion; i.e., the action verbs, the descriptive adjectives, and the denotative nouns relating objects commonly experienced together. The finding has obvious significance for theories providing for an early "syntactic" language learning as opposed to later "paradigmatic" development (Ervin, 1961; Entwisle et al, 1964).

\section{References}

Brown, R., \& Berko, Jean, Word association and the acquisition of grammar. Child Develpm., 1960, 31, 1-14.

Entwisle, Doris R., Forsyth, D. F., \& Muuss, R. The syntacticparadigmatic shift in children's word associations. J. verbal Learn. verbal Behav., 1964, 3, 19-29.

Ervin. Susan M. Changes with age in the verbal determinants of word-association. Amer. J. Psychol., 1961, 74, 361-372.

Riegel, K. F., Riegel, Ruth M., Smith, Helen E., \& Quarterman, Carole J. An analysis of differences in word meaning and semantic structure between four educational levels. Tech. RED. No. 2. Dept. Psychol., University of Michigan, 1964.

Watson, R. I. Psychology of the child. New York: Wiley, 1959. Woodworth, R. S. Experimental psychology. New York: Holt, 1938. Notes

1. Appreciation is extended to Dr. K. F. Riegel for his aid and comments.

2. Now at Brookwood Child Care Agency, New York City.

3. Now at Stanford University.

4. The complete coding scheme, with explanations, examples, and results by subdivision, is available from the junior author. 